\title{
Study of two-layered circular patch using moment method and genetic algorithms
}

\author{
Ahmed Mahamdi ${ }^{1}$, Siham Benkouda ${ }^{2}$, Mounir Amir ${ }^{3}$, Sami Bedra ${ }^{4}$ \\ ${ }^{1,2}$ LHS Laboratory, Department of Electronics, University of Mentouri Brothers - Constantine 1, Algeria \\ ${ }^{3}$ Department of Electronics, University Mouloud MAMMERI of Tizi-Ouzou, Algeria \\ ${ }^{4}$ Department of Industrial Engineering, University of Khenchela, Algeria
}

\begin{tabular}{l} 
Article Info \\
\hline Article history: \\
Received Dec 25, 2018 \\
Revised Jul 4, 2019 \\
Accepted Jul 17, 2019 \\
\hline
\end{tabular}

\section{Keywords:}

Accurate formula Genetic algorithm Moment method Patch resonator Two-layered

\begin{abstract}
In this paper, new expressions for the effective radius and fringing capacitance have been derived to predict accurately the resonant frequency of the two-layered circular microstrip patch antenna. These expressions are obtained based on genetic algorithm and the data base is generated using moment method (MOM). The proposed model is very simple, fast, and valid for an entire range of permittivities and thicknesses of two-layered substrate. The present model has been validated by comparing our numerical results obtained for the resonant frequencies with measurements. Finaly, the effect of the two-layered substrate on the resonant charateristics of the circular microstrip patch antenna has been presented.
\end{abstract}

Copyright $@ 2019$ Institute of Advanced Engineering and Science. All rights reserved.

\section{Corresponding Author:}

Siham Benkouda,

LHS Laboratory, Department of Electronics,

University of Mentouri Brothers - Constantine 1,

Constantine 25000, Algeria.

Email: siham.benkouda@umc.edu.dz

\section{INTRODUCTION}

Microstrip antennas have gained importance and created interest in research during these last years due to their numerous unique and attractive features such as low profile, light weight, planar configuration, relatively cheap, and ease in fabrication and integration with solid-state devices [1-5]. They have been employed as radiating or receiving elements in a wide range of microwave systems, such as radars, navigation, mobile communications [6] and wireless biotelemetry [7]. In microstrip antenna design, it is important to ascertain the resonant frequency of the antenna accurately because microstrip antennas have narrow bandwidths [8-12] and can only operate effectively in the vicinity of the resonant frequency. As such, a theory to help ascertain the resonant frequency is helpful in antenna designs.

The most popular methods used to compute the resonant frequency of microstrip antennas are the magnetic wall cavity model and the moment method implemented in the spectral domain known as the spectral domain approach. The cavity model is based on some fundamental simplifying physical assumptions regarding the radiation mechanism of antennas. This model results in simple analytical formulas, leading to a better understanding of the physical phenomenon, and well compatible with CAD. However, this model can lead to inaccurate results, especially for applications that require a thick substrate $[13,14]$ or a high dielectric constant. Another handicap specific to this model is its limitation to simple geometric shapes. 
The spectral domain approach is founded on the basis of complex mathematical formalisms, can lead to exploitable results only through a numerical resolution. This approach provides good accuracy but its computational cost is high due to the evaluation of the slowly decaying integrals and the iterative nature of the solution process. Hence, it is not easily included in a computer-aided design package.

The suspended substrate patch antenna has received a great deal of attention due to its frequency tenability feature, longer bandwidth, and improved radiation efficiency for a patch on high permittivity substrate (Alumina, GaAs, etc.). The suspended substrate patch antenna is a special type of a patch antenna on a two-layered substrate. The patch on a two-layered substrate is encountered in several microstrip designs [15].

A simple algebraic formula for the resonant frequency of a single-layer circular microstrip disk antenna as a function of the effective radius and of the fringing capacitance has been derived in [16] which is valid for electrically-thick dielectric substrates. In this paper, we propose fast and accurate model based on genetic algorithm in conjunction with moment method [13] to compute the resonant frequency of two-layered circular patch antenna. Genetic algorithm is used to obtain closed form expressions for the effective radius and the fringing capacitance by which the resonant frequency of the two-layered configuration may be easily calculated. This relatively simple model allows designers to predict accurately the resonant frequency for a given design without having to develop or run the spectral method codes themselves. The main advantages of the method are: less computing time than the spectral approach, results with accuracy equivalent to that of full-wave models and cost effectiveness, since the client can use a simple PC for implementation.

\section{GENETIC ALGORITHM : CONCEPTS}

Genetic algorithm is a method based on biological evolution. Basically, genetic algorithm has at least the following elements in common:

\subsection{Initial population}

In genetic algorithm based optimization, a set of trial solutions in the form of chromosomes is assembled as a population. The population is the unit that the genetic algorithm optimizer utilizes to search for the optimum solution [17]. The population size is chosen to achieve a compromise between computation time and solution quality.

\subsection{Evaluation}

The evaluation of each individual is performed by an evaluation function called fitness function. This function is usually the link between the physical problem and the genetic algorithm optimization process [17]. It's assigns to an individual a number representing the "goodness" of the trial solution represented by that individual.

\subsection{Selection}

Once the assessment is made of generation, it makes a selection from the fitness function. Only individuals passing the selection test can access the middle generation and breed (in our application we chose the selection by decimation).

\subsection{Crossover}

Its work is divided into two phases: the first is to randomly select the pairs to be crossed, the second, making the crossing after selecting a site randomly. Two new chromosomes are created, each with a portion of their "parents".

\subsection{Mutation}

The mutation is a mechanism for ensuring that overly aggressive selection does not result in premature convergence to a suboptimal solution. Mutation introduces the genetic material that is not present in the current population [17]. So this operator is simply the inversion of a bit in a chromosome.

\section{MOMENT METHOD PROCEDURE}

Consider a circular patch of radius $a$ printed on a two-layered grounded dielectric substrate as shown in Figure 1 . The first (second) layer of thickness $d_{1}\left(d_{2}\right)$ has a relative permittivity $\varepsilon_{r 1}\left(\varepsilon_{r 2}\right)$. All fields and currents are time harmonic with the $e^{\mathrm{i} \omega t}$ time dependence suppressed. Let $\mathbf{J}(\rho, \phi)=\left[\begin{array}{ll}J_{\rho}(\rho, \phi) & J_{\phi}(\rho, \phi)\end{array}\right]^{T}$ (where $T$ implies transpose) be the surface current density on the disc 
Also, let $\mathbf{E}(\rho, \phi)=\left[E_{\rho}(\rho, \phi) E_{\phi}(\rho, \phi)\right]^{T}$ be the value of the transverse electric field at the plane of the circular disc. Owing to the revolution symmetry of the two-layered medium of Figure 1 around the z-axis, when the Helmholtz equations for the longitudinal field components $E_{z}$ and $H_{z}$ are solved in cylindrical coordinates inside each of the two layers of that medium, it turns out that the dependence of $E_{z}$ and $H_{z}$ on the $\phi$ coordinate is of type $e^{\mathbf{i} k \phi}$ (where $k$ is an integer), as a consequence, $\mathbf{J}(\rho, \phi)$ and $\mathbf{E}(\rho, \phi)$ can be written as

$$
\begin{aligned}
& \mathbf{J}(\rho, \phi)=\sum_{k=-\infty}^{+\infty} e^{\mathbf{i} k \phi} \mathbf{J}_{k}(\rho) \\
& \mathbf{E}(\rho, \phi)=\sum_{k=-\infty}^{+\infty} e^{\mathbf{i} k \phi} \mathbf{E}_{k}(\rho)
\end{aligned}
$$

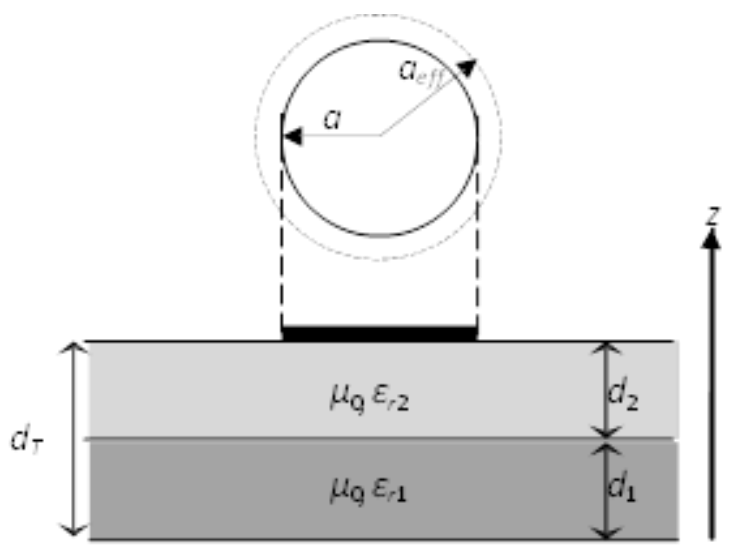

Figure 1. Geometry of a circular patch on a two-layered substrate

Following a mathematical reasoning similar to that shown in [13, 18-20], we obtain a relation among $\mathbf{J}(\rho, \phi)$ and $\mathbf{E}(\rho, \phi)$ in the spectral domain given by

$$
\mathbf{e}_{k}\left(k_{\rho}\right)=\overline{\mathbf{G}}\left(k_{\rho}\right) \cdot \mathbf{j}_{k}\left(k_{\rho}\right)
$$

where $\mathbf{j}_{k}\left(k_{\rho}\right)$ and $\mathbf{e}_{k}\left(k_{\rho}\right)$ are, respectively, the vector Hankel transforms of $\mathbf{J}_{k}(\rho)$ and $\mathbf{E}_{k}(\rho)$, and $\overline{\mathbf{G}}\left(k_{\rho}\right)$ stands for the dyadic Green's function. It is given by

$$
\overline{\mathbf{G}}\left(k_{\rho}\right)=\overline{\mathbf{L}}^{12} \cdot\left[\overline{\mathbf{L}}^{22}-\overline{\mathbf{g}}_{0} \cdot \overline{\mathbf{L}}^{12}\right]^{-1}
$$

where

$$
\overline{\mathbf{L}}=\overline{\mathbf{T}}_{2} \cdot \overline{\mathbf{T}}_{1}
$$

The matrix $\overline{\mathbf{T}}_{1}\left(\overline{\mathbf{T}}_{2}\right)$ is the matrix representation of the first (second) layer [4, in (7)]. Now that we have thje necessary Green's function, it is relatively straight forward to formulate the moment method solution for the antenna characteristics. The current on the circular disc is expanded into a finite series of known basis functions [21-24].

$$
\mathbf{J}_{k}(\rho)=\sum_{p=1}^{P} a_{k p} \boldsymbol{\Psi}_{k p}(\rho)+\sum_{q=1}^{Q} b_{k q} \boldsymbol{\Phi}_{k q}(\rho)
$$


$P$ and $Q$ correspond to the number of basis functions and $a_{k p}$ and $b_{k q}$ are the mode expansion coefficients to be sought. Substituting the vector Hankel transform of (6) into (3). Next, the resulting equation is tested by the same set of basis functions that was used in the expansion of the patch current. Thus, the integral equation is decomposed into the following matrix equation [13]:

$$
\overline{\mathbf{\Omega}}_{k} \cdot \mathbf{c}_{k}=\mathbf{0}
$$

where

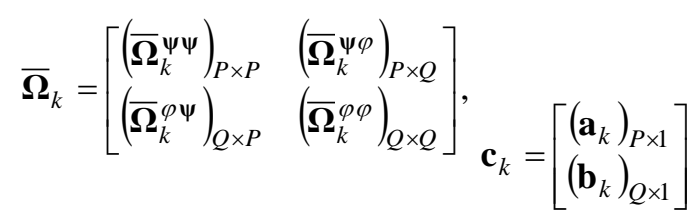

Each element of the submatrices of $\overline{\mathbf{\Omega}}_{k}$ is given by

$$
\overline{\mathbf{\Omega}}_{k}^{\mathbf{w w}}(i, j)=\int_{0}^{+\infty} \mathbf{v}_{k i}^{+}\left(k_{\rho}\right) \cdot \overline{\mathbf{G}}\left(k_{\rho}\right) \cdot \mathbf{w}_{k j}\left(k_{\rho}\right) k_{\rho} d k_{\rho}
$$

where $\mathbf{v}$ and $\mathbf{w}$ represent either $\psi$ or $\varphi$. For every value of the integer $k$, the system of linear (7) has nontrivial solutions when

$$
\operatorname{det}\left[\overline{\mathbf{\Omega}}_{k}(\omega)\right]=\mathbf{0}
$$

Equation (10) is an eigenequation for $\omega$, from which the resonant characteristics of the structure of Figure 1 can be obtained [13].

\section{NEW EXPRESSIONS FOR THE EFFECTIVE RADIUS AND FRINGING CAPACITANCE}

The resonant frequency of a microstrip patch antenna can be determined from the magnetic wall cavity model for various operational modes and structural parameters using proper equivalent model with effective structural parameters $[15,16]$. In the cavity model, the region between the patch and the ground plane can be considered as a cavity bounded by electric walls on the top and bottom, and by a magnetic wall on the side. The existence of fringing fields is taken into account by extending the edge slightly.

In this study, first the two-layered substrate is reduced to an equivalent single layer structure with total thickness $d_{T}=d_{1}+d_{2}$ and an equivalent relative permittivity of the form [2].

$$
\varepsilon_{r e q}=\frac{\varepsilon_{r 1} \varepsilon_{r 2}\left(d_{1}+d_{2}\right)}{\varepsilon_{r 1} d_{2}+\varepsilon_{r 2} d_{1}}
$$

The resonant frequency of the TM11 mode of the circular radiating element is given by

$$
f_{r}=\frac{27.6177}{\pi a_{e f f} \sqrt{\varepsilon_{d y n}}}
$$

Where $a_{e f f}$ is the effective patch radius and $\varepsilon_{d y n}$ stands for the dynamic permittivity. The expression of the effective patch radius used in [16] will be modified herin to account for the presence of a two-layered substrate in the micristrip structure under study:

$$
a_{\text {eff }}=\left\{a^{2}+\frac{2 d_{T} a}{\pi \varepsilon_{r}}\left[\ln \left(\frac{a}{2 d_{T}}\right)+\left(\beta_{1} \varepsilon_{e q}+\beta_{2}\right)+\frac{d_{T}}{a}\left(\beta_{3} \varepsilon_{e q}+\beta_{4}\right)\right]\right\}^{1 / 2}
$$


The dynamic permittivity of the equivalent single layer structure can be obtained in terms of the finging capacitances as follows:

$$
\varepsilon_{d y n}=\frac{C\left(a, d_{T}, \varepsilon_{e q}\right)}{C\left(a, d_{T}, \varepsilon_{0}\right)}
$$

with

$$
\begin{aligned}
& C\left(a, d_{T}, \varepsilon_{e q}\right)=\frac{\beta_{5} \varepsilon_{0} \varepsilon_{e q} \pi a^{2}}{d_{T}}+a \varepsilon_{0}\left[\ln \left(\frac{a}{2 d_{T}}\right)+\left(\beta_{1} \varepsilon_{e q}+\beta_{2}\right)+\frac{d_{T}}{a}\left(\beta_{3} \varepsilon_{e q}+\beta_{4}\right)\right] \\
& C\left(a, d_{T}, \varepsilon_{0}\right)=\frac{\beta_{5} \varepsilon_{0} \pi a^{2}}{d_{T}}+a \varepsilon_{0}\left[\ln \left(\frac{a}{2 d_{T}}\right)+\left(\beta_{1}+\beta_{2}\right)+\frac{d_{T}}{a}\left(\beta_{3}+\beta_{4}\right)\right]
\end{aligned}
$$

In (13), (15), and (16), $\beta_{1}, \beta_{2}, \beta_{3}, \beta_{4}$, and $\beta_{5}$ are the unknown parameters which may be determined from the minimization of the following cost function using genetic algorithms:

$$
\operatorname{Err}=\sum_{i=1}^{N}\left(\frac{f_{r i}-f_{r i}^{M O M}}{f_{r i}^{M O M}}\right)^{2}
$$

where $f_{r i}$ is the resonant frequency computed from (12) and $f_{r i}^{M O M}$ is the resonant frequency obtained using the moment method procedure detailed in section 3 .

\section{NUMERICAL RESULTS AND DISCUSSION}

In applying the moment method procedure to solve the electric field integral equation, it is advantageous to express the unknown patch current in terms of appropriate basis functions formed by the set of transverse magnetic and transverse electric modes of a cylindrical cavity of radius $a$ with magnetic side walls and electric top and bottom walls. These current modes which are non-zero only on the circular disc are given by

$$
\boldsymbol{\psi}_{k p}(\rho)=\left[\begin{array}{c}
J_{k}^{\prime}\left(\beta_{k p} \rho / a\right) \\
\frac{\mathrm{i} k a}{\beta_{k p} \rho} J_{k}\left(\beta_{k p} \rho / a\right)
\end{array}\right], \varphi_{k q}(\rho)=\left[\begin{array}{c}
\frac{-\mathrm{i} k a}{\alpha_{k q} \rho} J_{k}\left(\alpha_{k q} \rho / a\right) \\
J_{k}^{\prime}\left(\alpha_{k q} \rho / a\right)
\end{array}\right]
$$

In (18), $J_{k}($.$) is the Bessel function of the first kind of order k$, and the prime denotes differentiation with respect to the argument. $\boldsymbol{\psi}_{k p}(\rho)$ correspond to the TM cavity modes and $\varphi_{k q}(\rho)$ correspond to the TE cavity modes. The constants $\beta_{k p}$ and $\alpha_{k q}$ correspond to the $p$ th and $q$ th zeros of $J_{k}^{\prime}\left(\beta_{k p}\right)$ and $J_{k}\left(\alpha_{k q}\right)$, respectively. Note that the vector Hankel transform of the above basis functions can be easily obtained in closed-form. The summations over $p$ and $q$ in (6) are decided upon by the convergence of the resonant frequency value. We have found that small number of basis functions suffices to obtained good convergent results.

Using the genetic algorithm, the fitness function given in (17) is minimized to determine the coefficients $\beta_{1}, \beta_{2}, \beta_{3}, \beta_{4}$, and $\beta_{5}$. The optimum values of the unknown parameters are summarized in Table 1.

Table 1. The Optimum values of the unknown parameters.

\begin{tabular}{cccccc}
\hline Unknown parameters & $\beta_{1}$ & $\beta_{2}$ & $\beta_{3}$ & $\beta_{4}$ & $\beta_{5}$ \\
\hline Optimum Values & 1.484 & 1.625 & 0.491 & 1.595 & 0.893 \\
\hline
\end{tabular}


To confirm the correctness of the proposed approach described in this paper, our calculated results are compared with experimental data. Numerical results are obtained for the experiment reported in [25]. The patch radius is $a=5 \mathrm{~cm}$ and the top substrate has a relative permittivity $\varepsilon_{r 2}=2.32$. The bottom dielectric substrate is considered as an air layer. Table 2 summarized the computed resonant frequencies and those obtained by experiment. It is seen form this Table that our computed results agree very well the measured data. The maximum percentage error being less than $1 \%$.

Table 2. Comparison between the calculated resonant frequencies and the measured data

\begin{tabular}{cccc}
\hline \multirow{2}{*}{$d_{1}(\mathrm{~mm})$} & \multicolumn{2}{c}{ Resonant frequency $(\mathrm{GHz})$} & \multirow{2}{*}{ Percentage error $(\%)$} \\
\hline 0 & Measured & Calculated & 0.53 \\
0.5 & 1.128 & 1.134 & 0.23 \\
1 & 1.286 & 1.283 & 0.07 \\
\hline
\end{tabular}

In Figure 2, we present numerical results for the resonant frequencies and quality factors of a circular microstrip patch on a two-layered substrate. The radius of the circular disc is $a=6.35 \mathrm{~mm}$. The dielectric located just below the circular patch has a relative permittivity $\varepsilon_{r 2}=9.8$, while the one in contact with the ground plane has a relative permittivity $\varepsilon_{r 1}=2.5$. The obtained results in Figure 2 indicate that the increase of $d_{2}$ causes the decrease of the resonant frequency. The opposite behavior is observed for the quality factor. These behaviors can be explained by the fact that when $d_{2}$ grows, the effective permittivity of the dielectric medium under the patch increases.

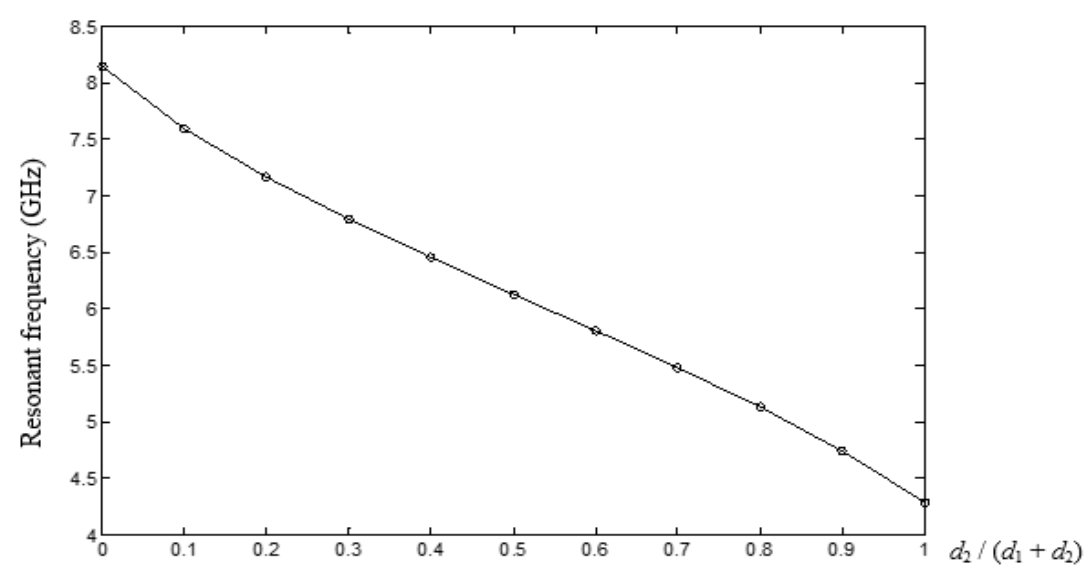

(a)

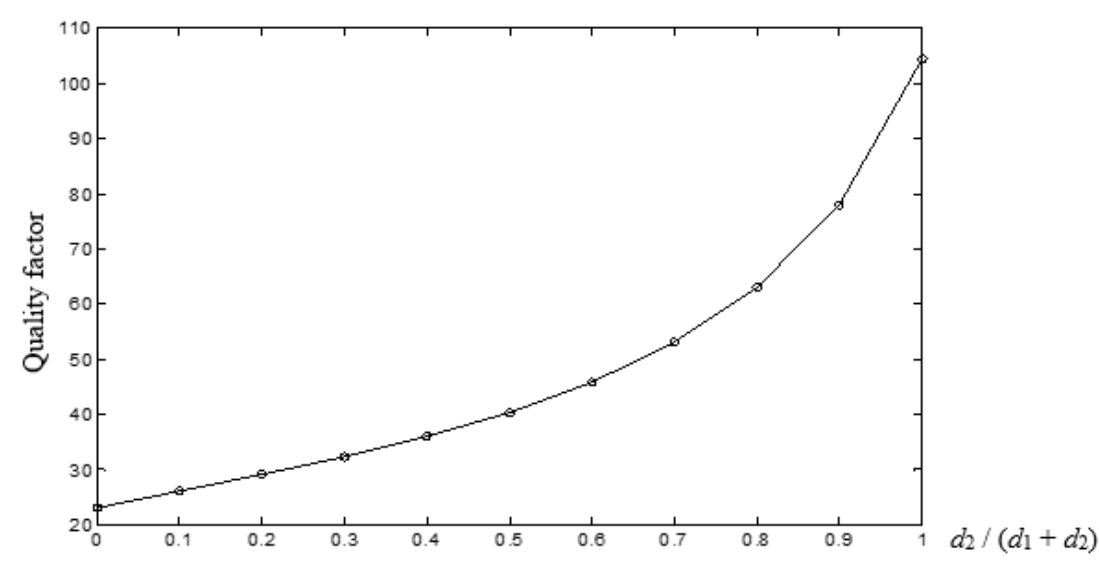

(b)

Figure 2. Resonant frequency and quality factor of the two-layered antenna; $a=6.35 \mathrm{~mm}, \varepsilon_{r 1}=2.5, \varepsilon_{r 2}=9.8$, $d_{1}+d_{2}=1 \mathrm{~mm}$. (a) Resonant frequency; (b) Quality factor 


\section{CONCLUSION}

The precise operating frequency of microstrip patch antenna is the most significant parameter to be determined in order to design such antenna system to achieve the optimum performance. This is because microstrip antennas have narrow bandwidth and hence can only operate efficiently in the vicinity of the resonant frequency. In this paper, a new model has been presented for the resonant frequency of a circular microstrip patch on a two-layered substrate. This model is well suited for computer aided design. It is based on genetic algorithms in conjunction with moment method. The present model is very simple, fast, accurate, and valid for an entire range of permittivities and thicknesses of two-layered substrate as well as a singlesubstrate patch antenna. The computed resonant frequencies have been compared with experimental values, and the present model shows very close agreements with the measured values. Finally, the effect of a twolayered substrate on the resonant characteristics of the circular disc microstrip antenna has been also investigated.

\section{REFERENCES}

[1] A. Messai, et al., "Analysis of High $T c$ Superconducting Rectangular Microstrip Patches Over Ground Planes with Rectangular Apertures in Substrates Containing Anisotropic Materials," International Journal of Antennas and Propagation, vol. 2013, pp. 1-7, 2013.

[2] S. Bedra, et al., "An Efficient Study of Circular Microstrip Antenna on Suspended and Composite Substrate," Journal of Computational Electronics, vol. 16, pp. 922-929, 2017.

[3] S. Bedra, et al., "Analysis of HTS Circular Patch Antennas Including Radome Effects," International Journal of Microwave and Wireless Technologies, vol. 10, pp. 843-850, 2018.

[4] N. Feiz, et al., "Optimized Microstrip Antennas with Metamaterial Superstrates Using Particle Swarm Optimization," Bulletin of Electrical Engineering and Informatics, vol. 2, pp. 123-131, 2013.

[5] N. Bahari, et al., "Gain Enhancement of Microstrip Patch Antenna Using Artificial Magnetic Conductor," Bulletin of Electrical Engineering and Informatics, vol. 8, pp. 166-171, 2019.

[6] N. Boukhennoufa, et al., "Effect of the Thickness of High Tc Superconducting Rectangular Microstrip Patch Over Ground Plane with Rectangular Aperture," International Journal of Electrical and Computer Engineering, vol. 8, pp. 1611-1617, 2018.

[7] M. H. Chowdhury, et al., "Single Feed Circularly Polarized Crescent-cut and Extended Corner Square Microstrip Antennas for Wireless Biotelemetry," International Journal of Electrical and Computer Engineering, vol. 9, pp. 1902-1909, 2019.

[8] Z. Er-reguig, et al., "A Miniature Broadband Microstrip Antenna for LTE, Wi-Fi and WiMax Applications," International Journal of Electrical and Computer Engineering, vol. 8, pp. 5238-5244, 2018.

[9] T. Firmansyah, et al., "Bandwidth Enhancement and Minaturization of Circular-shaped Microstrip Antenna Based on Beleved Half-cut Structure for MIMO 2x2 Application," International Journal of Electrical and Computer Engineering, vol. 9, pp. 1110-1121, 2019.

[10] D. Fitsum, et al., "Bandwidth Enhancement of Rectangular Microstrip Patch Antenna Using Defected Ground Structure," Indonesian Journal of Electrical Engineering and Computer Science, vol. 3, pp. 428-434, 2016.

[11] D. Fitsum, et al., "Dual-band Proximity Coupled Feed Microstrip Patch Antenna with 'T' Slot on the Radiating Patch and 'Dumbbell' Shaped Defected Ground Structure," Indonesian Journal of Electrical Engineering and Computer Science, vol. 3, pp. 435-440, 2016.

[12] T. Firmansyah, et al., "Bandwidth ang Gain Enhancement of MIMO Antenna by Using Ring and Circular Parasitic with Air-gap Microstrip Structure," TELKOMNIKA Telecommunication Computing Electronics and Control, vol. 15, pp. 1155-1163, 2017.

[13] M. Amir, et al., "Bacterial Foraging Optimisation and Method of Moments for Modelling and Optimisation of Microstrip Antennas," IET Microwaves, Antennas \& Propagation, vol. 8, pp. 295-300, 2014.

[14] S. Bedra, et al., "Efficient Full-wave Analysis of Inverted Circular Microstrip Antenna," Microwave and Optical Technology Letters, vol. 56, pp. 2422-2425, 2014.

[15] M. Biswas, et al., "Characteristics of Equilateral Triangular Patch Antenna on Suspended and Composite Substrate,” Electromagnetics, vol. 33, pp. 99-115, 2013.

[16] N. Kumprasert and W. Kiranon, "Simple and Accurate Formula for the resonant frequency of the Circular Microstrip disk Antenna," IEEE Transactions on Antennas and Propagation, vol. 43, pp. 1331-1333, 1995.

[17] R. L. Hopt and D. H. Werner, "Genetic Algorithms in Electromagnetic," New Jersey, John Wiley \& Sons, 2007.

[18] S. Bedra and T. Fortaki, "Hankel transform domain analysis of covered circular microstrip patch printed on an anisotropic dielectric layer," Journal of Computational Electronics, vol. 14, pp. 747-753, 2015.

[19] S. Bedra and T. Fortaki, "High-Tc superconducting rectangular microstrip patch covered with a dielectric layer," Physica C: Superconductivity and its Applications, vol. 524, pp. 31-36, 2016.

[20] T. Fortaki, et al., "Vector Hankel transform analysis of a tunable circular microstrip patch," International Journal for Numerical Methods in Biomedical Engineering, vol. 21, pp. 219-231, 2005.

[21] F. Benmeddour, et al., "Improving the performances of a high TC superconducting circular microstrip antenna with multilayered configuration and anisotropic dielectrics," PIER-Progress In Electromagnetics Research, vol. 18, pp. 169-183, 2010. 
[22] S. Bedra, et al., "Full-Wave Analysis of Anisotropic Circular Microstrip Antenna with Air Gap Layer," Progress In Electromagnetics Research, vol. 34, pp. 143-151, 2014.

[23] T. Fortaki, et al., "Study of High Tc Superconducting Microstrip Antenna," PIERS Online, vol. 5, pp. 346-349, 2009.

[24] S. Benkouda, et al., "Characteristics of a high Tc superconducting rectangular microstrip patch on uniaxially anisotropic substrate," Physica C: Superconductivity, vol. 502, pp. 70-75, 2014.

[25] J. S. Dahele and K. F. Lee, "Theory and Experiment on Microstrip Antennas with Airgaps," IEE Proceedings $H$ - Microwaves, Antennas and Propagation, vol. 132, pp. 455-460, 1985. 